\title{
Identification of hard-disk head actuator model using bacterial foraging- tabu search metaheuristics
}

\author{
Nuapett Sarasiri ${ }^{1}$, Siriwat Carawukh ${ }^{1,2}$, Vichitpon Boonpranchoo ${ }^{2}$, \\ Kishan Kumbla $^{3}$, and Sarawut Sujitjorn ${ }^{1 *}$ \\ ${ }^{1}$ School of Electrical Engineering, Institute of Engineering, Suranaree University of \\ Technology, Thailand \\ ${ }^{2}$ Hitachi Global Storage Technologies (Thailand), Prachinburi, Thailand \\ ${ }^{3}$ Hitachi Global Storage Technologies, San Jose, USA \\ *Corresponding author-Email: sarawut@sut.ac.th
}

\begin{abstract}
This short communication describes an industrial application of bacterial foraging-tabu search metaheuristics (BF-TS) to an identification problem of a hard-disk head actuator model. Using experimental data recorded from the actual systems, the model parameters can be obtained through a search-optimization process. The article presents the algorithm implementation, the identification and validation results.
\end{abstract}

Keywords: hard-disk head actuator, servo-track writing system, identification, bacterial foragingtabu search, metaheuristics.

\section{INTRODUCTION}

Hard disk drives (HDDs) are common in today's computers. A key process of HDD manufacturing to access data is the servo track writing (STW) process which provides servo patterns as references for the address of stored data. In STW process, there are two critical control problems: (i) problems caused by repeatable and non-repeatable run-out (RRO and NRRO) disturbances to the formation of servo patterns, and (ii) the misalignment of servo sectors occurred to the servo sectors of adjacent tracks (Mamun et. al., 2007). Self Servo Writing (SSW) (Brown et. al., 2000 and Dong et. al., 2006) has been introduced instead of conventional servo writing (Uematsu et. al., 2001). The SSW process can be represented by the diagram in Fig. 1 , in which the read/write heads write the servo patterns without using any external equipment. Some number of tracks called seed tracks are written on the disk first. The read head follows the seed tracks, and at the same time determine the track pitches, and the offsets between the read/write heads. The subsequent track is built until the corresponding servo patterns are written on the whole disk by referring to a previously written track or reference track. The SSW process is prone to problems caused by external disturbances, noises and resonances. To handle such problems by using control system technique, a control model of the hard-disk head actuator used for SSW is needed. The hard-disk head actuator means a set of $\mathrm{read} / \mathrm{write}$ head, arm, and voice-coil motor (VCM).

The next section presents the practical data, the model, and the algorithms. Results and discussions, and conclusion then follow in sequel.

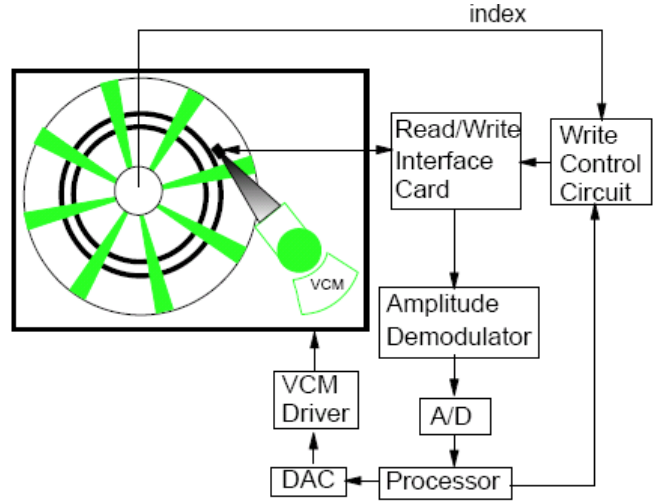

Fig 1 Block Diagram of SSW process.

\section{MATERIALS AND METHODS}

Referring to Fig. $2 \mathrm{a}$, it is noticed that the actuator dynamic in low frequency is dominated by the inertia that can be approximated by a double integrator. A torsional resonance mode clearly pronounces along side with butterfly and sway modes of resonance. 
Correspondingly, the s-domain model representing the dynamic can be expressed as
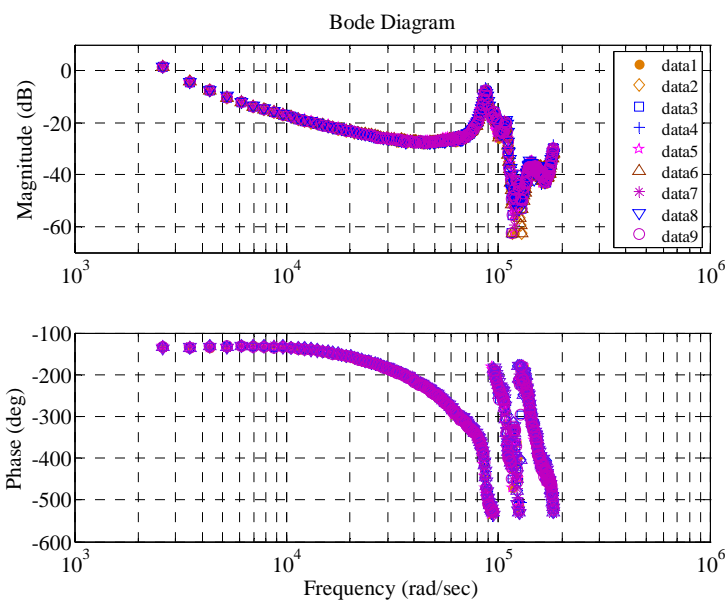

Fig 2a Frequency responses of the hard-disk head actuator obtained from measurement at the plant situated in Prachinburi, Thailand. The data are separated into 2 groups, i.e. $60 \%$ for identification, and $40 \%$ for validation purposes.
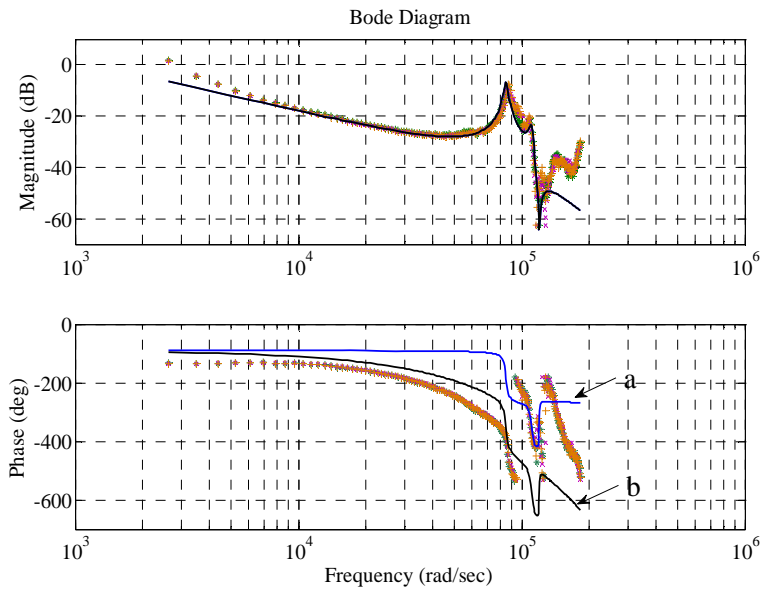

Fig 2 b Validation results. The scattered data represent the $40 \%$ of the measured data used for validation. The solid lines represent the model plots.

$G p(s)=\frac{K a}{J_{m} s^{2}}\left(\frac{\omega_{n 1}^{2}}{s^{2}+2 \varsigma_{n 1} \omega_{n 1} s+\omega_{n 1}^{2}}\right)\left(\frac{s^{2}+2 \varsigma_{n 3} \omega_{n 3} s+\omega_{n 3}^{2}}{s^{2}+2{\varsigma_{n 2}}_{n 2} s+\omega_{n 2}^{2}}\right)$

(1).

The order of the model is kept at minimum for simplification. The inertia of the actuator is known, $J_{m}=6.11 \times 10^{-7} \mathrm{~kg} \cdot \mathrm{m}^{2}$, the rest of the parameters need identification. Since the identification task is formulated as an optimization problem, we have applied the bacterial foraging-tabu search metaheuristics (BF-TS) as an optimization tool. The algorithms were successfully investigated for their efficiency over their predecessors (Panikhom et. al., 2010). The procedural lists of the algorithms are as follows:

Initialization of parameters: $p$, search space, $S$, $N_{C}, N_{S}, \alpha, d_{\text {attract }}, W_{\text {attract }}, h_{\text {repellant }}, w_{\text {repellant }}, R, N, T L$, count $_{\max }, \quad B T, \quad n \_r e \_b a c k$, best_neighbor and best_error. Randomly or heuristically select an initial solution within the search space and define as a set $\theta^{i}$.

while $j \leq N_{c}$ do

$$
j=j+1
$$

for each bacterium $i=1,2, \ldots, S$ do Compute $J(i, j)$

$$
\begin{aligned}
& J(i, j)=J(i, j)+J_{c c}\left(\theta^{i}(j), P(i, j)\right) \\
& J_{\text {last }}=J(i, j)
\end{aligned}
$$

Generate a $p$-dimensional random vector $\Delta_{p}(i) \in \mathfrak{R}^{p}, m=1,2, \ldots, p$ on $[-1,1]$. Compute the adaptive step size, $C(i)$

$$
\begin{aligned}
& C(i)=\frac{|J(i, j)|}{|J(i, j)|+\alpha}=\frac{1}{1+\frac{\alpha}{|J(i, j)|}} \\
& \theta^{i}(j+1)=\theta^{i}(j)+C(i, j) \frac{\Delta(i)}{\sqrt{\Delta^{\mathrm{T}}(i) \Delta(i)}}
\end{aligned}
$$

compute the objective function

$$
\begin{aligned}
& J(i, j+1)=J(i, j+1)+J_{c C}\left(\theta^{i}(j+1), P(j+1)\right) . \\
& J_{C C}\left(\theta^{i}, P(i, j)\right)=\sum_{i=1}^{s} J_{c C}^{i}\left(\theta, \theta^{i}(j)\right)
\end{aligned}
$$

where

$$
\begin{aligned}
= & \sum_{i=1}^{s}\left[-d_{\text {artract }} \exp \left(-w_{\text {artrac }} \sum_{m=1}^{p}\left(\theta_{m}-\theta_{m}^{i}\right)^{2}\right)\right] \\
& +\sum_{i=1}^{s}\left[h_{\text {repellen }} \exp \left(-w_{\text {repellen }} \sum_{m=1}^{p}\left(\theta_{m}-\theta_{m}^{i}\right)^{2}\right)\right]
\end{aligned}
$$

\footnotetext{
Let $m=0$

while $m<N_{S}$ do

$m=m+1$
} 


$$
\begin{aligned}
& \text { if } J(i, j+1)<J_{\text {last }} \text {, define } \\
& J_{\text {last }}=J(i, j+1) \text { and compute } \\
& \theta^{i}(j+1)=\theta^{i}(j+1)+C(i, j) \frac{\Delta(i)}{\sqrt{\Delta^{\mathrm{T}}(i) \Delta(i)}} \\
& \text { Use this } \theta^{i} \text { to compute the new } J(i, j+1) \\
& \text { else } \\
& m=N_{S} \\
& \text { end if } \\
& \text { end while } \\
& \text { end for } \\
& \text { end while }
\end{aligned}
$$

Do minimum sorting of the objective functions $\mathrm{J}$. Define best_ $\theta$ as the solution with the minimum $\mathrm{J}$. Set $S_{0}=$ best_ $\theta$.

for coun $\leq$ count $_{\max }$

- Generate a neighbourhood with an initial search radius $R$. Set $N$ solutions as the members of the set $S_{1}(r)$.

- Evaluate $J$ each member belonging to $S_{1}(r)$.

define $S_{1}=$ best_neighbor1 as a solution with the minimum cost, $J_{1}$.

if $J_{1}<J_{0}$ store $S_{0}$ in the $T L$

$$
\text { else }
$$$$
\text { define } S_{0}=S_{1}
$$

Store $S_{1}$ in the $T L$.

Invoke the $B T$ when a deadlock occurs.

BT: if $n \geq B T$

best_error $=$ RANK $(T L)$

look back in the $T L$, then retrieve the $n \_r e \_b a c k^{\text {th }}$ solution from the $T L$.

else

$n=0$

define $S_{0}=$ best_neighbor

best_error $=$ best_error

end if

Invoke the $A R$ when the current solution is close to local minima

AR: if best_error $<40$

$$
R=25
$$

end

if best_error $<25$

$R=5$

end

$\quad \begin{array}{r}R=0.5 \\ \text { end }\end{array}$
$\begin{aligned} & \text { end if } \\ & \text { end for }\end{aligned}$

For our identification problem, the gain $\mathrm{K}$ is defined as $K=\frac{K a}{J_{m}}$, search spaces and search parameters are assigned as follows:

$\left\{K=[0-1], \varsigma_{n 1}, \varsigma_{n 2}, \varsigma_{n 3}=[0-0.5]\right.$,

$\left.\omega_{n 1}=\left[8 \times 10^{4}-8.7 \times 10^{4}\right], \omega_{n 2}, \omega_{n 3}=\left[1 \times 10^{5}-1.2 \times 10^{5}\right]\right\}$,

$S=30, N_{C}=20, N_{S}=4, \alpha=0.001, d_{\text {attract }}, h_{\text {repellant }}=0.1$,

$w_{\text {attract }}=0.2, w_{\text {repellant }}=10, R=50, N=40$, count $t_{\max }=500$,

$B T=5$ and $n \_r e \_b a c k=5$.

The objective function $(J)$ representing a multiobjective requirement has a surrogate form of

$$
J=\left(0.2 \times \omega_{d 1_{\_} n}\right)+\left(0.2 \times \omega_{d 2_{-} n}\right)+\left(0.2 \times \omega_{d 3_{n} n}\right)+\left(0.2 \times J_{\text {gain }}\right)+\left(0.2 \times J_{\text {phase }}\right)
$$

Calculation of the objective function during the search process follows the steps listed below.

$$
\begin{aligned}
& \text { Function } \\
& \text { obj }\left(K, \omega_{n 1}, \varsigma_{n 1}, \omega_{n 2}, \varsigma_{n 2}, \omega_{n 3}, \varsigma_{n 3}\right) \\
& \text { Step1: Heuristically assign initial values for } \\
& \text { damped natural frequencies }(\mathrm{rad} / \mathrm{sec}) \text { based on test } \\
& \text { data. } \\
& \quad \omega_{d 1}=8.698 \times 10^{4} \quad, \quad \omega_{d 2}=1.087 \times 10^{5}, \\
& \omega_{d 3}=1.181 \times 10^{5} .
\end{aligned}
$$

Step2: Compute damped natural frequencies using natural frequencies and damping ratios proposed by search algorithms.

$$
\begin{aligned}
& \omega_{\mathrm{d} 1 \text { _iden }}=\omega_{n 1} \sqrt{1-\varsigma_{n 1}}, \omega_{\mathrm{d} 2 \text { _iden }}=\omega_{n 2} \sqrt{1-\varsigma_{n 2}} \\
& , \omega_{\mathrm{d} 3 \_ \text {iden }}=\omega_{n 3} \sqrt{1-\varsigma_{n 3}} .
\end{aligned}
$$

Step3: Accept solutions if the errors are bounded within $\pm 5 \%$, otherwise assign dummy values to the required solutions.

$$
\text { if } \omega_{\mathrm{d} 1 \_ \text {iden }}=\omega_{d 1} \pm 5 \% \text { then } \quad \omega_{\mathrm{d} 1 \_ \text {iden }}=\omega_{\mathrm{d} 1 \_ \text {iden }}
$$

else $\omega_{\text {d1_iden }}=1 \times 10^{10}$ end.

$$
\text { if } \quad \omega_{\mathrm{d} 2 \_ \text {iden }}=\omega_{d 2} \pm 5 \% \text { then } \quad \omega_{\mathrm{d} 2 \_ \text {iden }}=\omega_{\mathrm{d} 2 \_ \text {iden }}
$$

else $\omega_{\text {d2__den }}=1 \times 10^{10}$ end.

$$
\text { if } \omega_{\mathrm{d} 3 \_ \text {iden }}=\omega_{d 3} \pm 5 \% \text { then } \omega_{\mathrm{d} 3 \_ \text {iden }}=\omega_{\mathrm{d} 3 \_ \text {_den }}
$$

else $\omega_{\mathrm{d} 3 \_ \text {iden }}=1 \times 10^{10}$ end.

Step4: Normalize $\omega_{\mathrm{d} 1 \_ \text {iden }}, \omega_{\mathrm{d} 2 \_ \text {iden }}$ and $\omega_{\mathrm{d} 3 \_ \text {iden }}$. 


$$
\begin{aligned}
& \omega_{d 1_{-} n}=\omega_{d 1_{-} i d e n} / \omega_{d 1} \\
& \left.\omega_{d 2_{-} n}=\omega_{d 2_{-} i d e n} / \omega_{d 2}\right\} \text { must be within [0.95-1.05] } \\
& \omega_{d 3_{-} n}=\omega_{d 3_{-} i d e n} / \omega_{d 3}
\end{aligned}
$$

Step5: Compute RMS values of gain $(\mathrm{dB})$ and phase (deg).

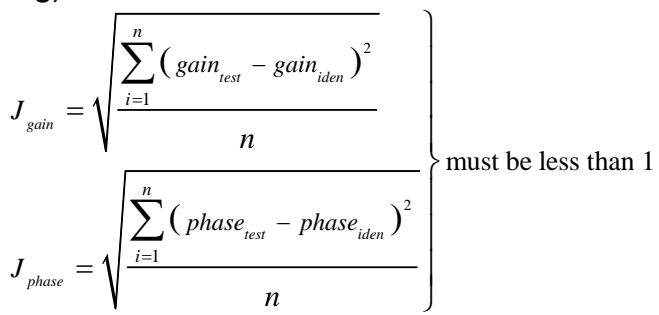

where $n=$ number of data points.

Step6: Compute the objective functions according to (2).

\section{RESULTS AND DISCUSSIONS}

To accept the search results, the cost value must meet the minimum threshold of 31.0.

Table 1 Parameters of the actuator model.

\begin{tabular}{|c|c|c|c|c|c|c|}
\hline$K$ & $S_{n 1}$ & $\omega_{n 1}$ & $\varsigma_{n 2}$ & $\omega_{n 2}$ & $\varsigma_{n 3}$ & $\omega_{n 3}$ \\
\hline 0.7673 & 0.0191 & $8.5006 \times 10^{4}$ & 0.0183 & $1.1087 \times 10^{5}$ & 0.0042 & $1.1984 \times 10^{5}$ \\
\hline
\end{tabular}

The $J_{\text {gain }}$ and $J_{\text {phase }}$ are computed as RMS errors of gain (dB) and phase (deg), which must be less than 1. The search was performed on the Intel(R) Core(TM)2Duo 2.00G Gz 1 Gbytes of RAM and took about 45 minutes. It stopped with the cost value of 30.8851. Referring to Fig $2 \mathrm{~b}$ for validation results, there are 2 modeled curves designated as "a" and "b". Curve "a" has a rather flat phase characteristic. Curve "b", when a delay of 40 micro-seconds is taken into account, better agrees with the measured phase data. This delay accounts for the processes of data sampling, conversion, and recording. However, both curves show considerable deviations from the measured phase data in high frequency range particularly where complex (anti)resonances presence. This is due to nonlinearity of the real actuator. Therefore, the "a" characteristics are accepted as an appropriate control model of the actuator to be used for control design. The corresponding model parameters are tabulated in table 1 . The $K$ value includes the actuator inertia $J_{m}=$ $6.11 \times 10^{-7} \mathrm{~kg} \cdot \mathrm{m}^{2}$.

\section{CONCLUSION}

Identification of the model parameters of a hard-disk head actuator in a self-servo writing system has been conducted based on frequency response data recorded from actual systems in the factory. The problem has been formulated as an optimization task, whereas the hybrid bacterial foraging optimization-tabu search algorithms or BF-TS metaheuristics have been applied as optimization algorithms. Successful search was performed based on the experimental data, terminated with the cost value of 30.8851 , and took 45 minutes approximately. Identification and validation results indicate a very satisfactory model obtained. The model will be further used for resonance compensation and control design.

\section{ACKNOWLEDGMENT}

The research grants supported by the HDD Institute, National Electronic and Computer Technology Center of Thailand (NECTEC) are greatly appreciated. The first and second authors are thankful to the Royal Golden Jubilee Ph.D. Program and HDDI-King Mongkut University of Technology Thonburi-NECTEC for their scholarships, respectively.

\section{REFERENCES}

Brown et al. (2000). Self Servo Writing File. U.S. Patent 6040955.

Dong, F.D., Ang, K. and Wang, Y.Y. (2006). TMR Analysis and Controller Design for Self-Servo Writing in HighDensity Hard Disk Drives. Proceedings of the International Conference on Control, Automation, Robotics and Vision (CDROM).

Panikhom, S., Sarasiri, N and Sujitjorn, S. (2010). Hybrid Bacterial Foraging and Tabu Search Optimization Algorithms for Lyapunov's Stability Analysis of Nonlinear Systems. International Journal of Mathematics and Computers in Simulation. 4(3):8189.

Mamun, A.A., Guo, G. and Bi, C. (2007). Hard Disk Drive: Mechatronics and Control-Automation and Control Engineering. CRC Press.

Uematsu, Y., Fukushi, M. and Taniguchi, K. (2001). Development of the Pushpin Free STW. IEEE Transactions on Magnetics, 37(2):964-968. 